\title{
Severe haematological toxicities in relapsed ovarian cancer treated with olaparib or niraparib: an Italian cost-minimization analysis
}

Global \& Regional Health Technology

Assessment

Volume 2019: 1-17

(C) The Author(s) 2019

Article reuse guidelines: sagepub.com/journals-permissions DOI: 10.1 | 777/228424031984394| journals.sagepub.com/home/grh @SAGE

\author{
Carlo Lazzaro' ${ }^{(D)}$, Nicola Amedeo Mazzanti² and Fabio Parazzini ${ }^{3}$
}

\begin{abstract}
The aim of this paper is to compare the cost for managing severe haematological toxicities (HTs: anaemia, neutropenia, thrombocytopenia) in patients with pretreated, relapsed BRCA-mutant positive $(+)$ ovarian cancer $(\mathrm{OC})$ receiving second-line therapy with olaparib or niraparib. As reported for treatment groups of randomized controlled trials, the incidence of anaemia, neutropenia and thrombocytopenia for olaparib (niraparib) was $0.07 \mathrm{I}, 0.432$ and $0.062(0.253$, 0.196 and 0.338 ), respectively. A one-year decision-tree cost-minimization analysis and a three-year budget impact analysis (BIA) were performed from the viewpoint of the Italian National Health Service (INHS). Health care resources funded by the INHS were identified and quantified based on the literature and expert opinion; they were costed via published sources and expressed in 2018 euro $(€)$ amounts on a per-patient basis. One-way sensitivity analysis (OWSA) and scenario analysis tested the robustness of the base case findings. Our results show that the costs for managing anaemia, neutropenia and thrombocytopenia with olaparib (niraparib) are $€ 440.63$, $€ 1032.76$ and $€|7| .0 I$ ( $€ I 568.82$, $€ 468.90$ and $€ 936.06$ ), respectively. The overall cost for $H T$ management reaches $€ \mid 644.4 \mathrm{I}$ and $€ 2973.78$ for olaparib and niraparib, respectively. Overall savings in favour of olaparib is $€$ I329.37. BIA shows that olaparib can save the INHS $€ 2.322 \mathrm{~m}$ over a three-year timespan. Sensitivity analyses confirm the robustness of the baseline findings. Despite some limitations in our study, the results of the cost-minimization analysis show that olaparib is a safe and cost-saving health care programme for the INHS for the management of HTs in patients receiving second-line therapy for BRCA+ OC.
\end{abstract}

\section{Keywords}

Ovarian cancer, haematological toxicities, olaparib, niraparib, cost-minimization analysis, Italy

\section{Introduzione}

In Italia, l'incidenza del carcinoma ovarico (CO) è pari a circa 5.200 nuovi casi/anno. ${ }^{1}$ Una percentuale compresa tra $80 \%$ ed il $90 \%$ dei casi di CO si presenta in pazienti di età compresa tra 20 e 65 anni, ${ }^{2}$ per le quali la sopravvivenza attesa a 5 e 10 anni dalla diagnosi sarà pari, rispettivamente, al $39 \%$ ed al $31 \% .{ }^{1}$ I CO si suddividono in neoplasie di tipo I e II. ${ }^{2}$ Le seconde, tra le quali si annovera il CO sieroso di alto grado, rappresentano circa il $70 \%-75 \%$ della totalità dei CO diagnosticati, ${ }^{2,3}$ sono caratterizzate da una marcata aggressività e spesso giungono all'osservazione del clinico in stato già avanzato. ${ }^{2}$

Mentre nella popolazione generale femminile il rischio di sviluppare un $\mathrm{CO}$ nel corso della propria vita è pari all' $1,8 \%,{ }^{2}$ tale percentuale aumenta dal $20 \%$ al $60 \%$ in presenza di mutazione nei geni $B R C A 1$ e $2,,^{2}$ diagnosticata mediante prelievo ematico (test germinale) o diagnosi del tessuto neoplastico (test somatico). ${ }^{4}$ Poli ADP-ribosio polimerasi (PARP) identifica una famiglia di proteine

\footnotetext{
IStudio di Economia Sanitaria, Milan, Italy

2Pricing \& Value Access Specialist Oncology, Market Access,

AstraZeneca S.p.A., Basiglio, Italy

${ }^{3}$ Dipartimento di Scienze Cliniche e di Comunità, Università di Milano, Milan, Italy

Corresponding author:

Carlo Lazzaro, Studio di Economia Sanitaria, Via Stefanardo da Vimercate, 19, Milan, I-20I28, Italy.

Email: carlo.lazzaro@tiscalinet.it
} 
attive deputate, tra l'altro, alla riparazione del DNA in seguito a danni a singolo filamento (single-strand breaks) e alla apoptosi cellulare. ${ }^{5}$ L'inibizione di PARP e del suo meccanismo di riparazione provoca la persistenza di danni a singolo filamento portando alla formazione di danni a doppio filamento, riparati dal sistema di ricombinazione omologa che viene guidato da $B R C A 1$ e 2 . Tuttavia, in cellule portanti mutazione $B R C A, B R C A 1$ e 2 non sono in grado di riparare il danno a doppio filamento causando la morte cellulare per accumulo di mutazioni. ${ }^{6}$

Olaparib (Lynparza ${ }^{\circledR}$; AstraZeneca, Londra, Regno Unito) è stato il primo PARP-inibitore (PARPi) a ottenere negli Stati Uniti d'America (USA) dalla Food and Drug Administration (FDA) l'indicazione per $\mathrm{CO}$ avanzato pretrattato $(\geqslant 3$ pregressi protocolli chemioterapici) con mutazione $B R C A^{7} \mathrm{e}$, più recentemente, anche per il trattamento del carcinoma della mammella localmente avanzato o metastatico human epidermal growth factor receptor 2-negativo nelle pazienti con mutazione $B R C A{ }^{8}$

Niraparib (Zejula ${ }^{\circledR}$; Tesaro, Waltham, MA, USA) è stato recentemente approvato per la terapia di mantenimento di pazienti adulte con $\mathrm{CO}$ epiteliale ricorrente, carcinoma delle tube di Falloppio o carcinoma peritoneale primario che abbiano ottenuto risposta completa o parziale dopo chemioterapia a base di platino. ${ }^{9}$

Per quanto attiene al profilo di tollerabilità, una caratteristica comune ai diversi PARPi è la frequenza di tossicità ematopoietiche (anemia, neutropenia, trombocitopenia), variabili in monoterapia o in associazione con chemioterapia, l'incidenza delle quali si qualifica, inoltre, per un'apprezzabile variabilità tra le diverse esperienze di ricerca. ${ }^{6,10}$ Tra le principali conseguenze cliniche delle tossicità ematopoietiche da PARPi, si annoverano la riduzione della posologia ${ }^{11}$ e la dilazione delle successive somministrazioni della terapia, ${ }^{12,13} \mathrm{con}$ possibili riflessi sulla gestione della neoplasia sottostante e, dunque, sulla sopravvivenza della paziente.

Sotto il profilo economico, due recenti esperienze di ricerca statunitensi ${ }^{12,14}$, hanno sottolineato che i costi unitari di gestione delle tossicità ematopoietiche da PARPi (espressi in US\$ a valori2017)variano traUS\$5.737 (trombocitopenia) e US\$13.633 (anemia), pari, rispettivamente, a euro $(€) 5.052,84$ e $€ 12.007,22$, (tasso di cambio $€ /$ US $\$=1,1354$, non aggiustato il tasso d'inflazione USA 2017-2018). ${ }^{15}$

\section{Obiettivo}

In assenza di pregressi contributi di ricerca relativi al nostro Paese, obiettivo del presente manoscritto è la descrizione della metodologia e dei risultati relativi al confronto tra le risorse sanitarie consumate per la gestione delle tossicità ematopoietiche, quali anemia, neutropenia e trombocitopenia ${ }^{6}$ di grado severo ( $>3$ secondo la vigente classificazione), ${ }^{16}$ quando si impiegano olaparib o niraparib nella terapia di seconda linea del $\mathrm{CO}$ recidivante con mutazione $B R C A$.

\section{Materiali e metodi}

\section{Tipologia della valutazione economica effettuata}

In quanto addendi di costo di trattamenti appartenenti alla medesima categoria terapeutica, caratterizzati da efficacia sovrapponibile sulla salute delle pazienti, ${ }^{17} \mathrm{i}$ costi delle tossicità ematopoietiche oggetto di indagine sono stati confrontati mediante analisi di minimizzazione dei costi, ${ }^{18,19}$ condotta secondo la prospettiva del Servizio Sanitario Nazionale (SSN). ${ }^{18,19}$

L'analisi di minimizzazione dei costi è stata supportata da un modello del tipo albero decisionale, ${ }^{19,20}$ caratterizzato da un singolo ciclo di durata annuale (Figure MSI \& MSII). Tale intervallo temporale è conservativo rispetto alla sopravvivenza libera da progressione di malattia (progression-free survival, PFS) mediana riportata in alcuni degli studi clinici randomizzati e controllati (randomized controlled trials, $\mathrm{RCTs}$ ) considerati nella presente ricerca. ${ }^{21,22}$

Posta la manifestazione di una delle tossicità ematopoietiche oggetto di indagine, l'albero decisionale, popolato da una serie di parametri separatamente dettagliati (Tabelle 1-3), considera due stati di salute esaustivi e mutuamente esclusivi: ${ }^{18,20,23}$ sopravvivenza (tale stato di salute rappresenta la condizione della paziente all'ingresso nel modello) e mortalità età-specifica per paziente affetta da $\mathrm{CO}^{24,25}$

Non è stata invece indagata l'evenienza della contemporanea presenza di due o più tipologie di tossicità ematopoietiche nella medesima paziente, ipotesi che avrebbe potuto generare economie di $\mathrm{scopo}^{26}$ nell'impiego delle risorse sanitarie necessarie alla loro gestione contestuale.

Nel calcolo dei costi si è applicata l'half-cycle correction, ${ }^{20,23}$ mediante la quale si ipotizza che il decesso della paziente avvenga a metà del ciclo di transizione (cioè a 6 mesi, nel caso in questione), anziché, alternativamente, all'inizio o al termine del medesimo, con riflessi sul calcolo del costo di gestione delle tossicità ematopoietiche totalizzato da olaparib e niraparib.

Il modello di analisi di minimizzazione dei costi è stato sviluppato su fogli di calcolo Microsoft ${ }^{\circledR}$ Excel $^{\circledR}$ per Windows ${ }^{\circledR} 2010$ (Microsoft Corporation, Redmond, WA, USA).

\section{Modalità raccolta dati}

Ad eccezione di alcune ipotesi di ricerca, i dati descritti ai paragrafi successivi sono stati ottenuti da fonti di letteratura, selezionate in collaborazione con il clinico co-autore del presente manoscritto. L'opinione qualificata del clinico ha inoltre consentito di confermare la plausibilità, con riferimento alla normale pratica clinica, della probabilità di utilizzo e del volume delle risorse sanitarie necessarie alla gestione degli eventi avversi di pertinenza ematologica considerati. 


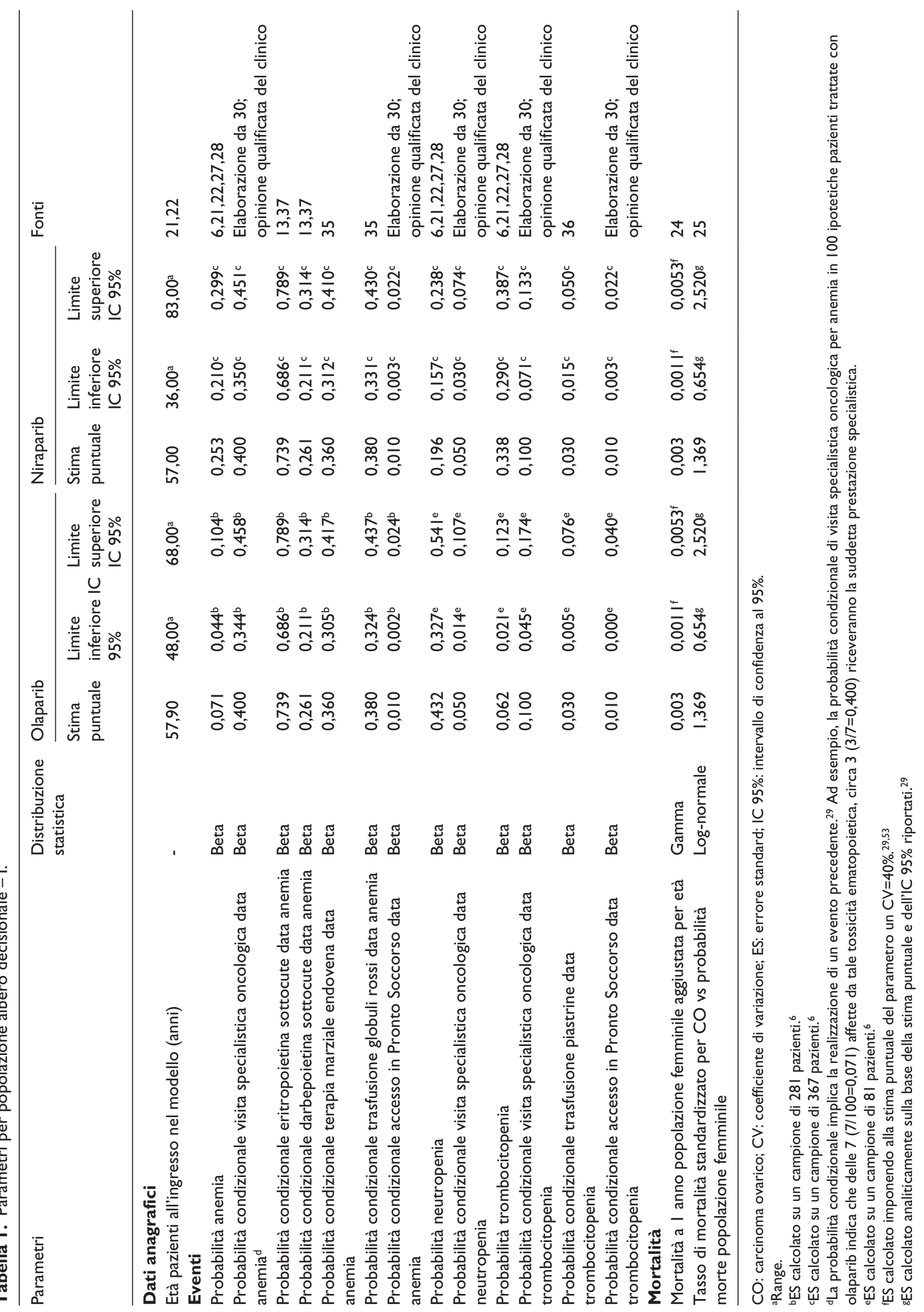




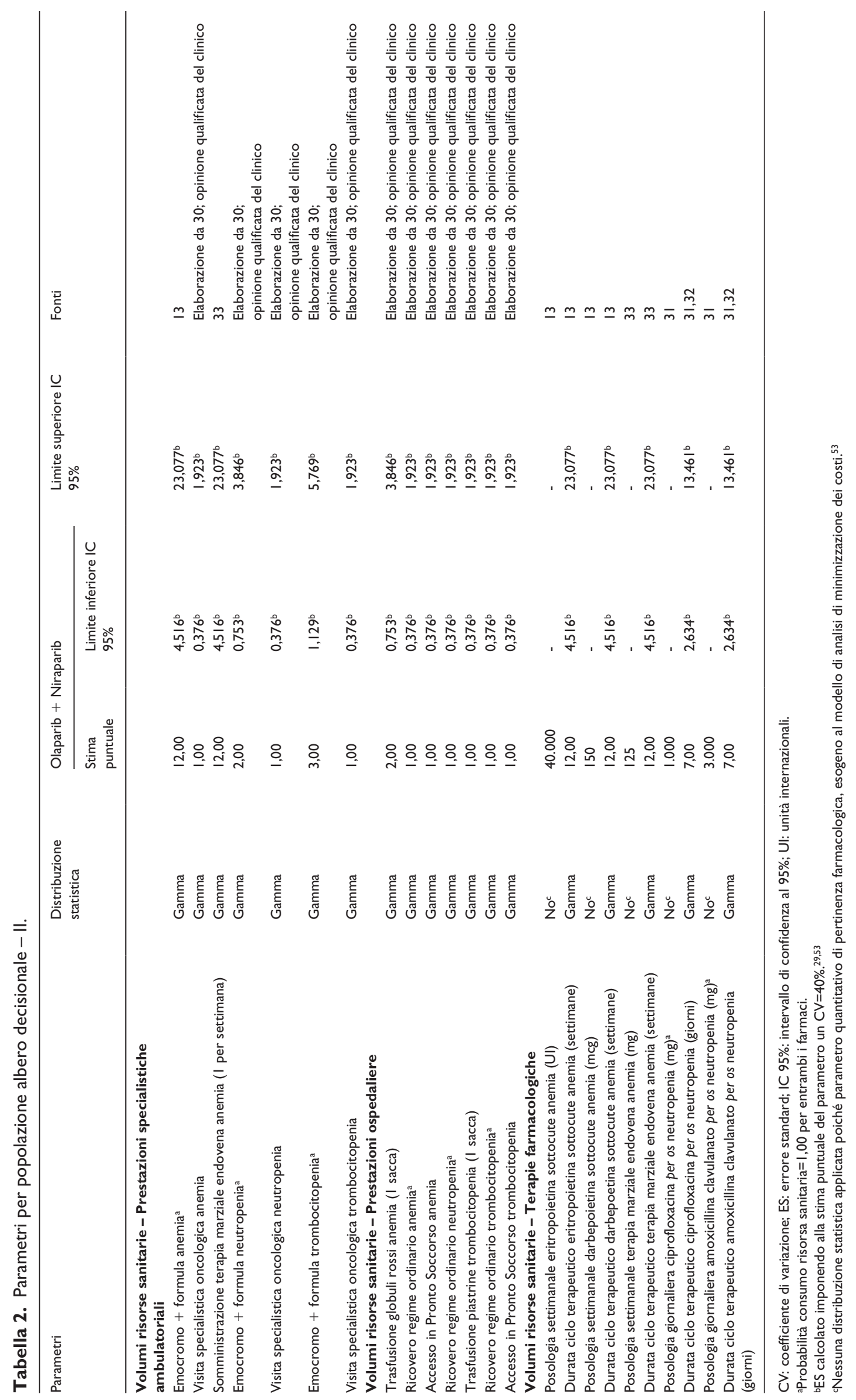


Tabella 3. Parametri per popolazione albero decisionale - III.

\begin{tabular}{|c|c|c|c|c|c|c|}
\hline \multirow[t]{2}{*}{ Parametri } & \multirow{2}{*}{$\begin{array}{l}\text { Distribuzione } \\
\text { statistica }\end{array}$} & \multicolumn{2}{|c|}{ Olaparib + Niraparib } & \multirow{2}{*}{$\begin{array}{l}\text { Limite superiore } \\
\text { IC } 95 \%\end{array}$} & \multirow{2}{*}{$\begin{array}{l}\text { Codice } \\
\text { prestazione }\end{array}$} & \multirow[t]{2}{*}{ Fonti } \\
\hline & & $\begin{array}{l}\text { Stima } \\
\text { puntuale }\end{array}$ & $\begin{array}{l}\text { Limite inferiore } \\
\text { IC } 95 \%\end{array}$ & & & \\
\hline \multicolumn{7}{|c|}{ Valorizzazione risorse sanitarie - Prestazioni specialistiche ambulatoriali $(€ 20 \mid 8)$} \\
\hline Emocromo + formula anemia & Normale & $€ 3,17$ & $€ 2,55^{\mathrm{a}}$ & $€ 3,79 a$ & 90.62 .2 & 40 \\
\hline Visita specialistica oncologica & Normale & $€ 20,66$ & $€|6,6|^{a}$ & $€ 24,7 I^{a}$ & 89.7 & 40 \\
\hline $\begin{array}{l}\text { Somministrazione terapia marziale } \\
\text { endovena ( } \mathrm{l} \text { per settimana) }\end{array}$ & Normale & $€ 10,30$ & $€ 8,28^{\mathrm{a}}$ & $€ 12,32^{\mathrm{a}}$ & 99.22 & 43 \\
\hline \multicolumn{7}{|c|}{ Valorizzazione risorse sanitarie - Prestazioni ospedaliere $(€ 20 \mid 8)$} \\
\hline $\begin{array}{l}\text { Confezionamento sacca globuli rossi } \\
\text { anemia }\end{array}$ & Normale & $€ 153,00$ & $€|23,0|^{\mathrm{a}}$ & $€ 182,99 a$ & 99736 & 41 \\
\hline $\begin{array}{l}\text { Trasfusione sacca globuli rossi anemia } \\
\text { (ricovero regime diurno) }\end{array}$ & Normale & $€ 134,00$ & $€ 107,74^{\mathrm{a}}$ & $€ 160,26^{\mathrm{a}}$ & DRG 467 & $4 I$ \\
\hline Ricovero regime ordinario anemia & Normale & $€ 652,00$ & $€ 524,21^{\mathrm{a}}$ & $€ 160,26^{a}$ & DRG 467 & 41 \\
\hline Ricovero regime ordinario neutropenia & Normale & $€ 2.357,71$ & $€ 1.895,6 \mathrm{I}^{\mathrm{a}}$ & $€ 2.8|9,8|^{a}$ & DRG 398; 399b & 41,42 \\
\hline Ricovero regime diurno trombocitopenia & Normale & $€ 2.748,00$ & $€ 2.209,40^{a}$ & $€ 3.286,60^{a}$ & DRG 397 & 41 \\
\hline $\begin{array}{l}\text { Confezionamento sacca piastrine } \\
\text { trombocitopenia }\end{array}$ & Normale & $€|| 5,00$ & $€ 92,46^{\mathrm{a}}$ & $€ 137,54^{\mathrm{a}}$ & 99744 & 41 \\
\hline $\begin{array}{l}\text { Trasfusione sacca piastrine } \\
\text { trombocitopenia (ricovero regime } \\
\text { diurno) }\end{array}$ & Normale & $€ 271,00$ & $€ 217,88^{a}$ & $€ 324,12^{\mathrm{a}}$ & DRG 397 & 41 \\
\hline Accesso in Pronto Soccorso & Normale & $€ 24 I, 05$ & $€|93,8|^{a}$ & $€ 288,30^{a}$ & - & 39 \\
\hline \multicolumn{7}{|c|}{ Valorizzazione risorse sanitarie - Terapie farmacologiche $(€ 20 \mid 8)$} \\
\hline Eritropoietina sottocute anemia (UI) & Noc & $€ 0,010$ & - & - & - & 38 \\
\hline Darbepoietina sottocute anemia (mcg) & Noc & $€ 3,23$ & - & - & - & 38 \\
\hline Terapia marziale endovena anemia (mg) & Noc & $€ 0,27$ & - & - & - & 38 \\
\hline Terapia marziale per os anemia (mg) & Noc & $€ 0,002$ & - & - & - & 38 \\
\hline Ciprofloxacina per os neutropenia & Noc & $€ 0,002$ & - & - & - & 38 \\
\hline $\begin{array}{l}\text { Amoxicillina clavulanato per os } \\
\text { neutropenia }\end{array}$ & Noc & $€ 0,00 \mathrm{I}$ & - & - & - & 38 \\
\hline
\end{tabular}

CC: complicanze e comorbilità; CV: coefficiente di variazione; DRG: Diagnosis-related group; ES: errore standard; IC 95\%: intervallo di confidenza al 95\%; SDO: scheda di dimissione ospedaliera; Ul: unità internazionali.

${ }^{\mathrm{a} E S}$ calcolato imponendo alla stima puntuale del parametro un $\mathrm{CV}=10 \% .29,53$

bLa tariffa DRG in oggetto è il risultato della somma di ciascuna tariffa DRG per neutropenia (DRG 398 - Disturbi del sistema reticoloendoteliale ed immunitario con CC: €3.589,00; DRG 399 - Disturbi del sistema reticoloendoteliale ed immunitario senza CC: $€$ I.704,00), ${ }^{41}$ moltiplicata per la proporzione delle dimissioni da SDO relative a ciascun codice DRG (DRG 398: 2.602; DRG 399: 4.90I) sul totale delle dimissioni da SDO inerenti a tale tossicità ematopoietica codificate mediante i DRG 398 e 399 nel 2016.42 La tariffa DRG ponderata consegue pertanto dal seguente calcolo: $\{€ 3.589,00 *[2.602 /(2.602+4.90 \mathrm{I})]\}+\{€ \mathrm{I} .704,00 *[4.90 \mathrm{I} /(2.602+4.90 \mathrm{I})]\}=€ 2.357,7 \mathrm{I}$.

`Nessuna distribuzione statistica applicata poiché parametro quantitativo monetario di pertinenza regolatoria, esogeno al modello di analisi di minimizzazione dei costi. ${ }^{53}$

La collaborazione con il clinico si è articolata in una serie di quattro interviste de visu avvenute nei mesi di Agosto-Settembre 2018.

\section{Incidenza delle tossicità ematopoietiche}

Da una recente meta-analisi realizzata su 12 RCTs condotti su 2.479 pazienti affetti da diverse neoplasie (CO recidivante con mutazione $B R C A$ [5 RCTs]; carcinoma polmonare non a piccole cellule (non small-cell lung cancer, NSCLC) [2 RCTs]; carcinoma della mammella [2 RCTs]; melanoma [1 RCT]; tumore gastrico metastatico [1 RCT]; carcinoma polmonare a piccole cellule [1 RCT] sottoposti a terapia con PARPi ${ }^{6}{ }^{6}$ è stata identificata l'incidenza delle tossicità ematopoietiche sopra citate per olaparib ${ }^{21,27,28}$ e niraparib ${ }^{22}$ con esclusivo riferimento al $\mathrm{CO}$ pretrattato recidivato con mutazione $B R C A$.

Per olaparib, le incidenze degli eventi avversi ematologici sono state ricavate da tre RCTs per quanto riguarda l'anemia ${ }^{21,27,28}$ e da un unico RCT per quanto concerne neutropenia e trombocitopenia (Tabella 1). ${ }^{21}$

Le probabilità di anemia, che differivano nei tre RCTs, risultavano pari a 0,086 (7/81 pazienti) assumendo olaparib $200 \mathrm{mg}$ bid in combinazione con chemioterapia (docetaxel + carboplatino) verso chemioterapia, ${ }^{21} 0,094$ (6/64 pazienti) a seguito di somministrazione di olaparib 200 o $400 \mathrm{mg}$ in monoterapia due volte al giorno (bid) verso doxorubicina pegilata liposomiale ${ }^{27}$ e $0,051(7 / 136$ pazienti) con olaparib $400 \mathrm{mg}$ in monoterapia bid verso placebo. ${ }^{28}$ 
Il valore medio della probabilità di anemia nelle pazienti trattate con olaparib $(0,071)$ è stato calcolato ponderando le stime puntuali sopra indicate per il numero di pazienti avviate a olaparib in ciascuna delle tre fonti considerate, diviso per la somma di tali dimensioni campionarie $(81 / 281,64 / 281$ e 136/281, rispettivamente) (Tabella 1$)$.

Sempre per olaparib, l'incidenza di neutropenia e trombocitopenia risultavano pari a 0,432 (o $35 / 81$ pazienti) e 0,062 (o 5/81 pazienti), rispettivamente ${ }^{21}$ (Tabella 1 ).

Con riferimento a niraparib, l'incidenza delle tre tossicità ematopoietiche (anemia: 0,253 o 93/367 pazienti; neutropenia: 0,196 o 72/367 pazienti; trombocitopenia: 0,338 o $124 / 367$ pazienti) era riportata nel medesimo RCT, che prevedeva, nel confronto con placebo, la somministrazione, in monoterapia di mantenimento, della posologia di principio attivo per la quale esistono evidenze di efficacia, pari a $300 \mathrm{mg}$ una volta al giorno. ${ }^{22}$

\section{Identificazione e quantificazione delle risorse}

A motivo della prospettiva adottata nel modello di analisi di minimizzazione dei costi, sono state considerate esclusivamente le risorse di pertinenza del settore sanitario $^{19}$ finanziate dal SSN, consumate per la gestione delle tossicità ematopoietiche, a proposito delle quali sono state definite, unitamente a identità e volume, le probabilità condizionali ${ }^{29}$ di utilizzo (Tabelle 1 e 2).

Le risorse sanitarie comprendono accertamenti laboratoristici ambulatoriali (limitati ad emocromo + formula, nell'ipotesi che ulteriori parametri ematici e bioumorali siano invece monitorati in regime di ricovero ordinario); $; 0$ farmaci a somministrazione sottocutanea per gestione in contesto extraospedaliero di anemia (eritropoietina; darbepoietina) ${ }^{13}$ e neutropenia (amoxicilina/ clavulanato e ciprofloxacina per os), ${ }^{31,32}$ farmaci a somministrazione endovenosa in regime ambulatoriale per anemia (esclusivamente per terapia marziale); ${ }^{33}$ visite specialistiche oncologiche; ${ }^{30}$ confezionamento sacche di emazie (anemia) e di piastrine (trombocitopenia) e relativa trasfusione in regime di ricovero diurno; ${ }^{34-36}$ ospedalizzazione inregimeordinario(anemia, neutropeniaetrombocitopenia); 30 accessi in Pronto Soccorso (anemia e trombocitopenia) ${ }^{30}$ (Tabelle 1 e 2).

La probabilità di consumo di eritropoietina $\mathrm{o}$ darbepoietina, prescritte quali alternative alle totalità delle pazienti con anemia, ${ }^{13}$ è stata determinata costruendo un rapporto avente al denominatore la somma delle più recenti defined daily dose (DDD) per 1.000 abitanti di epoetina alfa $(1,7)$ e darbepoietina $(0,6)$ ed al numeratore la DDD per 1.000 abitanti della prima. ${ }^{37}$ Applicando tale procedimento, si è stimato che la probabilità di prescrizione di epoetina alfa fosse pari a $0,739(1,7 / 2,3)$, ricavando, quale complemento all'unità, la probabilità di prescrizione per darbepoietina, pari a 0,261 (cioè: 1-0,739).
Poiché per la totalità degli studi considerati ${ }^{21,22,27,28}$ non risultava una chiara distinzione tra incidenza di neutropenia febbrile e non febbrile, si è considerata quest'ultima, ipotizzando la profilassi antibiotica verso il rischio di evoluzione febbrile. ${ }^{31,32}$

Ancora con riferimento alla neutropenia, non sono stati considerati i fattori di crescita mieloide, poiché né indicati in pazienti con neutropenia non febbrile,,$^{13}$ né raccomandati in associazione ad antibioticoterapia. ${ }^{13,32}$

Per quanto concerne l'ospedalizzazione, si è assunto che il ricovero in regime ordinario fosse finalizzato, alternativamente, alla valutazione polispecialistica volta alla diagnosi differenziale delle possibili cause sottostanti a ciascuna tossicità ematopoietica conseguente alle terapie oncologiche, o all'effettuazione di idonei approfondimenti laboratoristici o strumentali, nei casi in cui l'attivazione delle restanti risorse sanitarie non risultasse sufficiente alla risoluzione della problematica clinica. In particolare, all'interno dell'episodio di ricovero ordinario, è stata ipotizzata l'esecuzione di almeno una delle seguenti procedure: esofagogastroduodenoscopia e colonscopia (anemia); somministrazione di ciclo di terapia antibiotica a somministrazione esclusivamente ospedaliera (es.: vancomicina endovena) ${ }^{32}$ in aggiunta a terapia antifungina e antivirale $^{32}$ (neutropenia); ecografia addome completo e biopsia osteomidollare (trombocitopenia).

\section{Valorizzazione delle risorse}

I costi di pertinenza del settore sanitario sostenuti dal SSN includono la totalità delle risorse sanitarie consumate per la gestione delle tossicità ematopoietiche esaminate.

I farmaci sono stati valorizzati mediante il prezzo al pubblico, ${ }^{38}$ mentre le restanti prestazioni sanitarie, erogate in ambito ambulatoriale od ospedaliero (regime diurno ed ordinario; emergenza ed urgenza) sono state monetizzate impiegando i riferimenti tariffari più recenti di libera consultazione $^{39-43}$ (Tabella 3). Tutte le voci di costo considerate, calcolate per paziente, sono espresse in $€$ a valori 2018.

Poiché il confronto tra olaparib e niraparib non eccede l'anno, coerentemente a quanto stabilito da linee-guida internazionali e nazionali sulla valutazione economica dei programmi sanitari, ${ }^{18,19,44}$ i costi unitari di gestione delle tossicità ematopoietiche non sono stati oggetto di attualizzazione.

\section{Determinazione di impatto budgetario}

La determinazione di impatto budgetario ${ }^{45,46}$ sulle disponibilità economico-finanziarie del SSN di durata triennale (2018-2020), conseguente alla prescrizione di olaparib invece di niraparib, si è focalizzata sulle pazienti affette da $\mathrm{CO}$ pretrattato recidivato $B R C A+$ diagnosticate 
in Italia, per le quali sussiste indicazione al trattamento con entrambi i PARPi comparati. $7,9,47,48$

Pertanto, le pazienti eleggibili a olaparib sono state stimate isolando, dai casi di $\mathrm{CO}$ incidenti annui, ${ }^{2,49}$ la tipologia di CO sieroso ad alto grado in seconda linea di trattamento con ricaduta platino-sensibile, risposta completa o parziale alla chemioterapia a base di platino e mutazione $B R C A^{1,3,4,49-52}$ (Tabella 4).

Sono state inoltre adottate le seguenti ipotesi di ricerca:

- permanenza degli assunti e dei risultati del modello di analisi di minimizzazione dei costi;

- assenza di impedimenti di carattere clinico e/o vincoli logistici al passaggio delle pazienti da niraparib a olaparib;

- assenza di attualizzazione dei costi manifestatisi nel secondo e terzo anno compresi nel modello di determinazione di impatto budgetario. ${ }^{46}$

\section{Analisi statistica}

L'analisi statistica si è limitata alla determinazione, per la maggioranza dei parametri utilizzati nel modello di analisi di minimizzazione dei costi e nella determinazione di impatto budgetario, dell'IC 95\%, calcolato utilizzando alcune distribuzioni statistiche codificate in letteratura, ${ }^{20,53}$ quali Beta (probabilità eventi dicotomici), Gamma (volumi risorse sanitarie, a esclusione della posologia dei farmaci, e mortalità a un anno popolazione generale aggiustata per età), Normale (costi unitari risorse sanitarie, ad esclusione dei farmaci) e Log-normale (moltiplicatore mortalità per $\mathrm{CO}$ rispetto a mortalità a un anno popolazione generale aggiustata per età) (Tabelle 1-3).

Quando non determinato analiticamente, l'errore standard della distribuzione campionaria della media di ciascun parametro, necessario per la determinazione dell'IC95\%, è stato calcolato imponendo, alla stima puntuale utilizzata nell'analisi di base, un coefficiente di variazione ${ }^{29}$ raccomandato dalla letteratura (volumi risorse sanitarie $=40 \%$; costi unitari risorse sanitarie $=10 \%)^{53}$ (Tabelle 1-3).

L'analisi statistica è stata supportata dal software Excel per Windows ${ }^{\circledR} 2010$ (Microsoft, Redmond, WA, USA).

\section{Analisi di sensibilità}

La robustezza dei risultati ottenuti con l'analisi di base è stata verificata mediante due tipologie di analisi di sensibilità: univariata e, per quanto concerne la sola analisi di minimizzazione dei costi, di scenario. ${ }^{18,19,54}$

Analisi di sensibilità univariata. L'incertezza relativa alla stima puntuale dei parametri che hanno popolato il modello di analisi di minimizzazione dei costi è stata indagata mediante l'analisi di sensibilità univariata, ${ }^{18,19,54}$ la quale prevede il cambiamento di un singolo parametro per volta, mantenendo i restanti costanti, cioè al medesimo valore impiegato nell'analisi di base.

L'analisi di sensibilità univariata si è orientata alla sostituzione della stima puntuale della maggioranza dei parametri utilizzati nel modello con i limiti inferiori e superiori dei rispettivi IC 95\% o range di variazione (Tabelle 1-3).

I risultati dell'analisi di sensibilità univariata sono stati riportati su un grafico Tornado, ${ }^{18}$ gli assi cartesiani del quale si incrociano in prossimità della differenza tra i costi complessivi per paziente di olaparib e niraparib risultanti dall'analisi di base.

Per quanto concerne la determinazione di impatto budgetario, si sono sostituiti gli estremi dell'IC 95\% della distribuzione Beta alla stima puntuale della probabilità di manifestazione degli eventi che concorrono alla definizione del numero delle pazienti eleggibili a olaparib (Tabella 4).

Analisi di sensibilità di scenario. Nell'analisi di sensibilità di scenario, ${ }^{54}$ le probabilità di manifestazione delle tossicità ematopoietiche utilizzate nell'analisi di base, ${ }^{6}$ mantenute invariate, sono state moltiplicate per i costi unitari di gestione dei medesimi eventi avversi di grado $\geqslant 3$ riportati in due recenti esperienze di ricerca condotte nel nostro Paese, riferite a due differenti neoplasie (adenocarcinoma del pancreas ${ }^{30}$ e NSCLC con mutazione epidermal growth factor receptor, $\mathrm{EGFR}^{55}$ ).

In entrambi gli studi, le risorse sanitarie necessarie alla gestione delle tossicità ematopoietiche sono state identificate e quantificate sulla base dell'opinione degli esperti (raccolta mediante un sondaggio realizzata presso nove centri oncologici ${ }^{30}$ o pannelli Delphi che ha coinvolto tre clinici esperti55) e monetizzate in $€ 2017$ secondo la prospettiva del SSN.

Le analisi di sensibilità sono state supportate dal software Excel per Windows ${ }^{\circledR} 2010$ (Microsoft, Redmond, WA, USA).

\section{Risultati}

\section{Analisi di minimizzazione dei costi}

Analisi di base. I costi unitari relativi alla gestione delle tossicità ematopoietiche indagate sono pari ad €6.190,92 (anemia), $€ 2.390,11$ (neutropenia) e $€ 2.770,43$ (trombocitopenia) (Tabella 5).

Per entrambi i PARPi comparati, i costi di pertinenza del SSN relativi alle suddette tossicità ematopoietiche sono stati calcolati moltiplicando i rispettivi costi unitari per la probabilità di manifestazione di tali eventi avversi (Tabelle 1 e 5).

Nel corso di un anno, olaparib e niraparib presentano i costi totali per paziente minori $(€ 1.644,41)$ e maggiori (€2.973,78), rispettivamente (Tabella 5). 


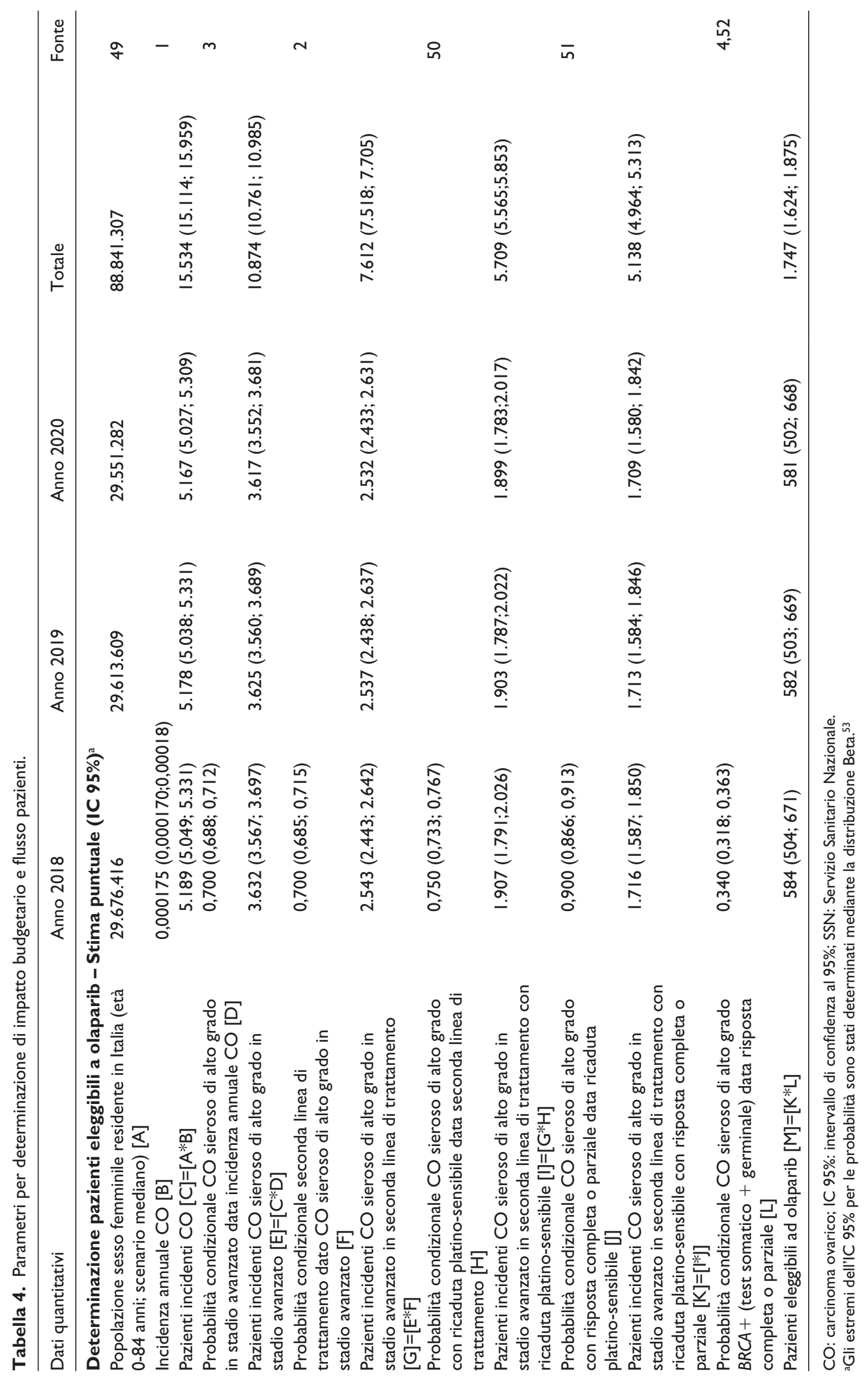




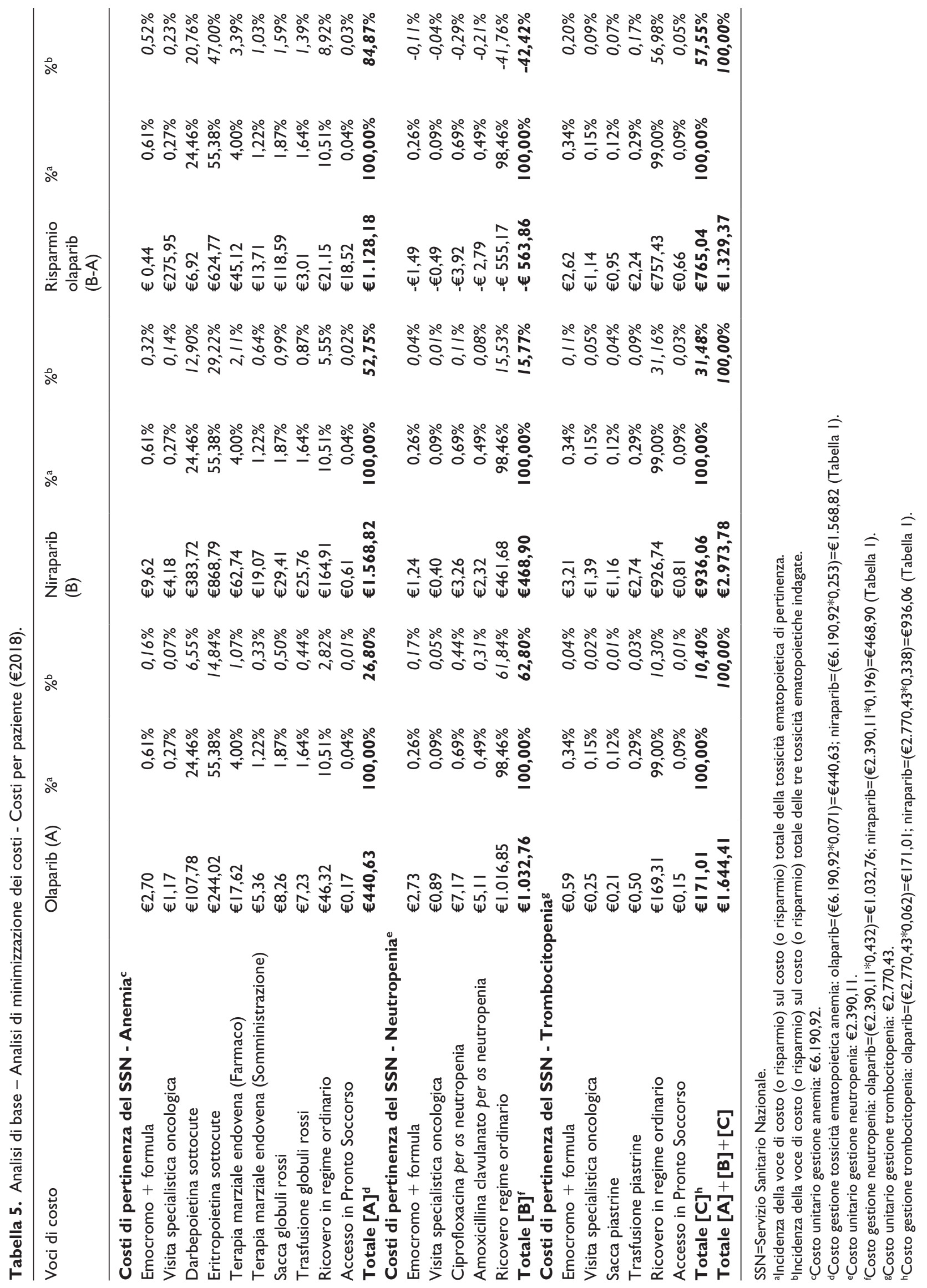




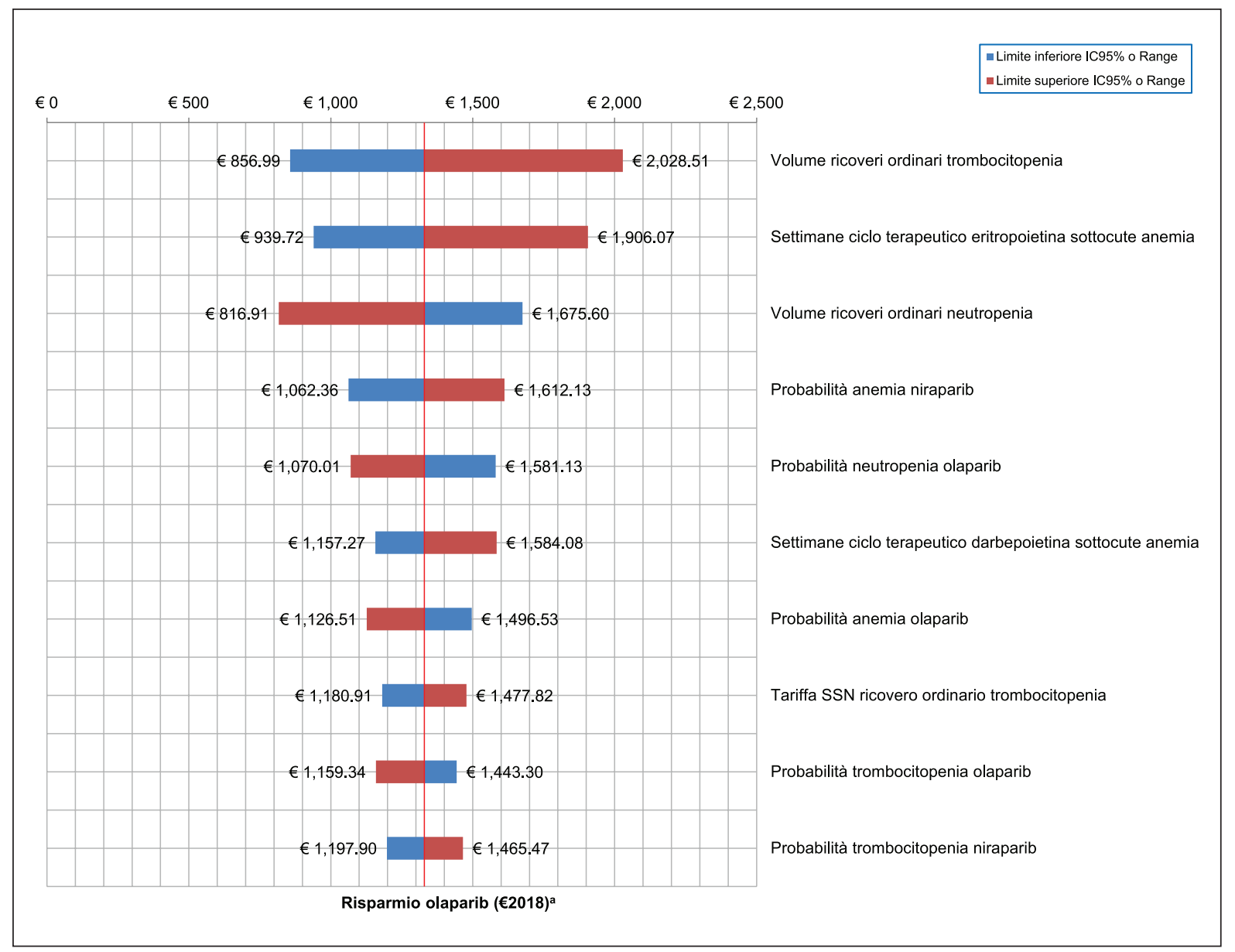

Figura I. Analisi di sensibilità univariata - Analisi di minimizzazione dei costi - Risparmi per paziente (€20।8). aRisparmio olaparib analisi di base $=€ 1.329,37$.

IC 95\%: intervallo di confidenza al 95\%; SSN: Servizio Sanitario Nazionale.

Per quanto concerne olaparib (niraparib), la tossicità ematopoietica che genera i maggiori costi per paziente a carico del SSN, pari a $€ 1.032,76(€ 1.568,82)$ è rappresentata dalla neutropenia (anemia), che incide per il $62,80 \%$ $(52,75 \%)$ sul costo totale per paziente.

Per converso, l'effetto avverso di pertinenza ematologica ascrivibile a olaparib (niraparib) che comporta i costi minori per il terzo pagante, che eguagliano $€ 171,01$ (€468,90), pari al $10,40 \%(15,77 \%)$ del costo totale per paziente, si identifica nella trombocitopernia (neutropenia).

Per entrambe le molecole, i driver dei costi delle singole tossicità ematopoietiche sono rappresentati dall'eritropoietina sottocute $(55,38 \%$ del costo totale per paziente relativo all'anemia) e dal ricovero in regime ordinario $(98,46 \%$ e $99,00 \%$ del costo totale per paziente, relativo alla neutropenia ed alla trombocitopenia, rispettivamente).

$\mathrm{Nel}$ corso di un anno, il risparmio complessivo per paziente a favore di olaparib risulta pari a $€ 1.329,37$; 1 ' $84,87 \%$ di tale importo deriva dai minori costi per paziente totalizzati da tale principio attivo per la gestione dell'anemia.
Analisi di sensibilità univariata. Limitando la descrizione dei risultati ai 10 parametri che determinano le maggiori variazioni rispetto all'analisi di base, il risparmio per paziente a favore di olaparib risulta moderatamente sensibile alla sostituzione della stima puntuale con i limiti dell'IC 95\% del volume di ricoveri in regime ordinario per trombocitopenia (da $-35,53 \%$ a $+52,59 \%$ vs analisi di base) e del numero di settimane di terapia con eritropoietina in caso di anemia (da $-29,31 \%$ a $+43,38 \%$ vs analisi di base) (Figura 1 ).

Anche a motivo della minore dispersione intorno alla stima puntuale dei parametri, i riferimenti utilizzati per la monetizzazione delle risorse sanitarie necessarie alla gestione delle tossicità ematopoietiche indagate, rivestono un ruolo trascurabile nella verifica della maggiore efficienza economica di olaparib determinata con le ipotesi di partenza. Si discostano da tale tendenza i limiti dell'IC 95\% della valorizzazione del ricovero in regime ordinario per trombocitopenia (da $-11,17 \%$ a $+11,17 \%$ vs analisi di base).

Producono, infine, diminuzioni e aumenti più contenuti, variazioni indotte nella probabilità di trombocitopenia sia 
per olaparib ( $\mathrm{da}-12,79 \% \mathrm{a}+8,57 \%$ vs analisi di base), che per niraparib (da $-9,89 \%$ a $+10,24 \%$ vs analisi di base).

Analisi di sensibilità di scenario. Con riferimento all'anemia, i costi unitari di gestione variano da $€ 293,80$ ad $€ 469,21$, ampiamente inferiori al valore di $€ 6.190,92$ da noi calcolato (Tabella 6).

La neutropenia non febbrile assorbe risorse per un valore compreso tra $€ 12,50$ e $€ 205,40$, anche in questo caso assai inferiore a quanto determinato nel modello di analisi di minimizzazione dei costi dettagliato alle pagine precedenti (€2.390,11).

Infine, per la trombocitopenia gli importi oscillano tra $€ 145,72$ ed $€ 4.241,19$; quest'ultimo valore risulta, invece, notevolmente superiore a quanto calcolato nella presente esperienza di ricerca $(€ 2.770,43)$.

Complessivamente, i costi totali a carico del SSN a motivo delle tossicità ematopoietiche indagate, sono compresi tra $€ 131,08$ e $€ 289,21$ per olaparib (da $-92,03 \%$ a $-82,41 \%$ vs analisi di base) e €208,22 e €1.510,30 per niraparib (da $-92,30 \%$ a $-49,21 \%$ vs analisi di base)

Indipendentemente dalla fonte utilizzata nell'analisi di sensibilità di scenario, il risparmio a favore di olaparib permane positivo, anche se caratterizzato da evidente variabilità, sia in termini assoluti (da €77,14 a €1.221,09), sia in termini di variazione percentuale (da $-94,20 \%$ a $-8,15 \%$ vs analisi di base).

\section{Determinazione di impatto budgetario}

Analisi di base. Nel corso del triennio ipotizzato dal modello di determinazione di impatto budgetario, 1.747 (IC 95\%: $1.624 ; 1.875)$ pazienti incidenti risultano eleggibili a terapia con olaparib (Tabella 7). Date le previsioni in diminuzione dello scenario mediano della popolazione femminile, che condiziona coerentemente il numero di nuovi casi di CO diagnosticati per anno, il numero delle pazienti eleggibili a olaparib segue una tendenza decrescente, ed è compreso tra 584 (IC 95\%: 504;671) pazienti (anno 2018) e 581 (IC 95\%: 502;668) pazienti (anno 2020).

In termini percentuali, il numero annuale e complessivo delle pazienti eleggibili a olaparib non eccede 1'11,25\% del totale dei casi incidenti di CO diagnosticati.

Il risparmio aggregato annuale a favore del SSN, generato dai minori costi per la gestione delle tossicità ematopoietiche totalizzati da olaparib, dato il previsto calo delle pazienti, si riduce contestualmente e si attesta intorno a $€ 0,776 \mathrm{~m}$ (anno 2018), €0,774m (anno 2019) e $€ 0,772 \mathrm{~m}$ (anno 2020), mentre il risparmio aggregato triennale raggiunge $€ 2,322 \mathrm{~m}$.

Analisi di sensibilità univariata. A seguito della sostituzione degli estremi inferiore e superiore dell'IC 95\% della distribuzione Beta relativa alla proporzione delle pazienti incidenti affette da $\mathrm{CO}$ sieroso di alto grado in stadio avanzato in seconda linea di trattamento, con ricaduta platino-sensibile, risposta completa o parziale con mutazione $B R C A$ (test somatico + germinale) eleggibili a olaparib, il trattamento con tale principio attivo consentirebbe, nella prima ipotesi, risparmi aggregati per il SSN pari ad $€ 2,234 \mathrm{~m}$ (-3,79\% vs all'analisi di base), mentre, nella seconda ipotesi, risparmi aggregati per il SSN pari ad $€ 2,411 \mathrm{~m}$ $(+3,83 \%$ rispetto all'analisi di base) (Tabella 7$)$.

\section{Discussione e conclusioni}

Il presente manoscritto ha descritto la metodologia e i risultati di un'analisi di minimizzazione dei costi ${ }^{18,19}$ condotta secondo la prospettiva del SSN, finalizzata alla comparazione dei costi totalizzati, nel corso di un anno, per la gestione delle tossicità ematopoietiche derivanti dalla terapia con olaparib o niraparib nelle pazienti affette da $\mathrm{CO}$ recidivato con mutazione $B R C A$.

L'intervallo temporale adottato, confortato dai risultati di PFS relativi ai PARPi confrontati, ${ }^{21,22}$ ha consentito di supportare l'analisi di minimizzazione dei costi mediante un albero decisionale ${ }^{20}$ semplice, caratterizzato da due stati di salute (sopravvivenza o decesso), che si aggiungono alla presenza/assenza delle tossicità ematopoietiche oggetto di indagine. È interessante rilevare che la PFS è raccomandata come endpoint primario (ritenuto maggiormente informativo della sopravvivenza totale a partire dalla terza linea di trattamento) negli studi clinici aventi per oggetto l'efficacia dei PARPi nel $\mathrm{CO}$, poiché questi includono pazienti che, pretrattate con diversi regimi chemioterapici, rappresentano, di fatto, una popolazione eterogenea. ${ }^{56}$

Pur con le note cautele che debbono caratterizzare il confronto con studi di carattere economico sanitario condotti all'estero, ${ }^{57}$ a motivo, ad esempio, delle differenze nelle modalità di valorizzazione delle risorse sanitarie, $i$ costi unitari di gestione delle medesime tossicità ematopoietiche rilevati nei due studi statunitensi precedentemente citati, ${ }^{12,14}$ espressi in US\$ a valori 2017, risultano costantemente superiori a quelli determinati nella presente esperienza di ricerca (anemia: US\$7.533-US\$7.680, pari ad €6.634,67-€6.764,14; neutropenia: US\$13.248-US\$13.633, pari ad €11.668,13US\$12.007,22; trombocitopenia: US\$5.737-US\$10.607, pari ad $€ 5.051,08-€ 9.342,08$ ) (tasso di cambio $€ / U S \$=1,1354$, non aggiustato per il tasso d'inflazione USA 2017-2018). ${ }^{15}$

Dai risultati descritti ai paragrafi precedenti emergono alcune indicazioni che si reputano interessanti per il decisore. In merito alla gestione delle tossicità ematopoietiche esaminate, nell'analisi di base e per la totalità delle ipotesi considerate nelle analisi di sensibilità univariata e di scenario, olaparib genera risparmi a favore del SSN. Pare opportuno sottolineare che i suddetti risparmi sono spiegati unicamente dalle differenti probabilità di manifestazione delle tossicità ematopoietiche oggetto di indagine, poiché le risorse sanitarie necessarie alla loro gestione sono state identificate, quantificate e valorizzate in maniera analoga per olaparib e niraparib. 


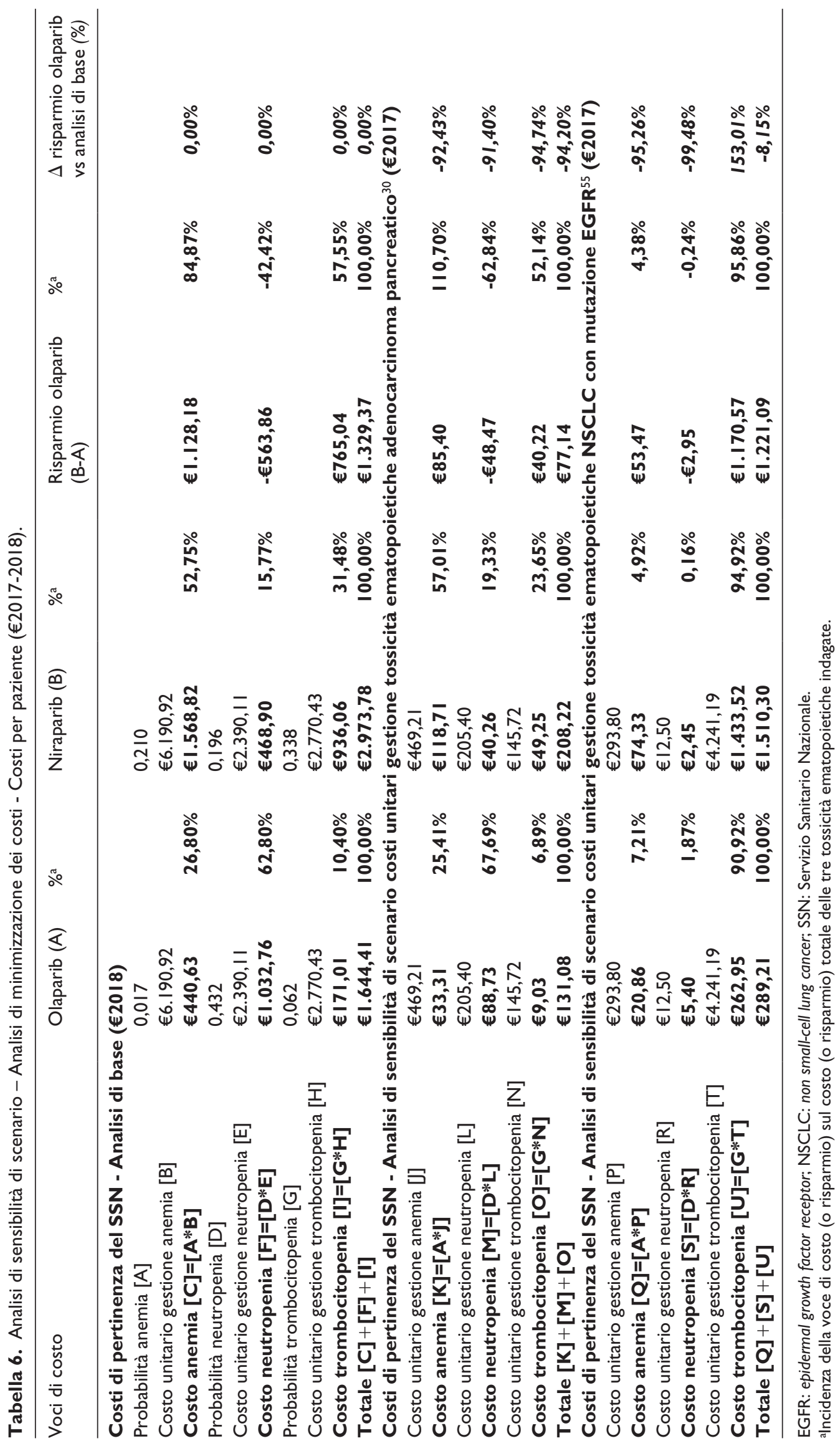


Tabella 7. Analisi di base e di sensibilità univariata - Determinazione di impatto budgetario - Risparmi aggregati SSN (€m 20I8) .

\begin{tabular}{|c|c|c|c|c|}
\hline Dati quantitativi & Anno 2018 & Anno 2019 & Anno 2020 & Totale \\
\hline \multicolumn{5}{|c|}{ Numero pazienti - Stima puntuale (IC 95\%) } \\
\hline Pazienti eleggibili a olaparib & $584(504 ; 67 I)$ & $582(503 ; 669)$ & $58 \mathrm{I}(502 ; 668)$ & $\mathrm{I} .747(\mathrm{I} .624 ; \mathrm{I} .875)$ \\
\hline \multicolumn{5}{|c|}{ Determinazione di impatto budgetario - Analisi di base } \\
\hline Risparmi aggregati SSN & $€ 0,776$ & $€ 0,774$ & $€ 0,772$ & $€ 2,322$ \\
\hline
\end{tabular}

Determinazione di impatto budgetario - Analisi di sensibilità univariata ( $\Delta$ vs analisi di base) - IC $95 \%$ incidenza annuale $\mathbf{C O}$

$\begin{array}{lllll}\begin{array}{l}\text { Risparmi aggregati SSN - Limite } \\ \text { inferiore IC 95\% }\end{array} & € 0,755(-2,70 \%) & € 0,753(-2,71 \%) & € 0,752(-2,71 \%) & € 2,259(-2,70 \%) \\ \begin{array}{l}\text { Risparmi aggregati SSN - Limite } \\ \text { superiore IC 95\% }\end{array} & € 0,797(+2,74 \%) & € 0,797(+2,96 \%) & € 0,794(+2,74 \%) & € 2,386(+2,74 \%)\end{array}$
superiore IC $95 \%$

Determinazione di impatto budgetario - Analisi di sensibilità univariata ( $\Delta$ vs analisi di base) - IC $95 \%$ probabilità condizionale $\mathbf{C O}$ sieroso di alto grado in stadio avanzato data incidenza annuale $\mathbf{C O}$

Risparmi aggregati SSN - Limite

$€ 0,762(-1,79 \%)$

$€ 0,760(-1,79 \%)$

$€ 0,759(-1,80 \%)$

$€ 2,298(-1,03 \%)$

inferiore IC 95\%

Risparmi aggregati SSN - Limite

$€ 0,789(+\mathrm{I}, 77 \%)$

$€ 0,788(+1,77 \%)$

$€ 0,786(+\mathrm{I}, 77 \%)$

$€ 2,346(+1,03 \%)$

superiore IC 95\%

Determinazione di impatto budgetario - Analisi di sensibilità univariata ( $\Delta$ vs analisi di base) - IC $95 \%$ probabilità condizionale seconda linea di trattamento dato $\mathrm{CO}$ sieroso di alto grado in stadio avanzato

Risparmi aggregati SSN - Limite

$€ 0,759(-2,14 \%) \quad € 0,757(-2,15 \%)$

$€ 0,756(-2,15 \%)$

$€ 2,294(-1,24 \%)$

inferiore IC 95\%

Risparmi aggregati SSN - Limite

superiore IC 95\%

$€ 0,792(+2,11 \%) \quad € 0,790(+2,12 \%)$

$€ 0,789(+2,12 \%)$

$€ 2,35 \mathrm{I}(+\mathrm{I}, 23 \%)$

Determinazione di impatto budgetario - Analisi di sensibilità univariata ( $\Delta$ vs analisi di base) - IC $95 \%$ probabilità condizionale $\mathrm{CO}$ sieroso di alto grado con ricaduta platino-sensibile data seconda linea di trattamento
Risparmi aggregati SSN - Limite
$€ 0,758(-2,27 \%)$
$€ 0,756(-2,27 \%)$
$€ 0,755(-2,27 \%)$
$€ 2,292(-I, 31 \%)$

inferiore IC 95\%

Risparmi aggregati SSN - Limite

$€ 0,793(+2,22 \%)$

$€ 0,79 \mid(+2,22 \%)$

$€ 0,790(+2,22 \%)$

$€ 2,352(+1,29 \%)$

superiore IC $95 \%$

Determinazione di impatto budgetario - Analisi di sensibilità univariata ( $\Delta$ vs analisi di base) - IC $95 \%$ probabilità condizionale $\mathrm{CO}$ sieroso di alto grado con risposta completa o parziale data ricaduta platino-sensibile

Risparmi aggregati SSN - Limite $€ 0,764(-I, 54 \%)$ $€ 0,762(-1,54 \%)$

$€ 0,76 \mid(-I, 54 \%)$

$€ 2,302(-0,88 \%)$

inferiore IC 95\%

Risparmi aggregati SSN - Limite

$€ 0,787(+\mathrm{I}, 45 \%)$

$€ 0,785(+\mathrm{I}, 45 \%)$

$€ 0,784(+\mathrm{I}, 45 \%)$

$€ 2,342(+0,85 \%)$

superiore IC $95 \%$

Determinazione di impatto budgetario - Analisi di sensibilità univariata ( $\Delta$ vs analisi di base) - IC $95 \%$ probabilità condizionale $\mathrm{CO}$ sieroso di alto grado BRCA + (test somatico + germinale) data risposta completa o parziale

Risparmi aggregati SSN - Limite inferiore IC 95\%

Risparmi aggregati SSN - Limit

superiore IC $95 \%$

$€ 0725(-6,54 \%)$

$€ 0,723(-6,54 \%)$

$€ 0,722(-6,55 \%)$

$€ 2,234(-3,79 \%)$

$€ 0,827(+6,64 \%)$

$€ 0,826(+6,65 \%)$

$€ 0,824(+6,65 \%)$

$€ 2,4 \mathrm{II}(+3,83 \%)$

CO: carcinoma ovarico; IC 95\%: intervallo di confidenza al 95\%; SSN: Servizio Sanitario Nazionale.

al risparmi aggregati riportati sono il risultato della moltiplicazione tra pazienti calcolati sulla base dei parametri relativi alla determinazione di impatto budgetario (Tabella 4) e il risparmio per paziente a favore di olaparib, pari a $€ 1.329,37$ (Tabella 5).

Vi è inoltre da considerare che, secondo alcuni Autori, ${ }^{10}$ in questa indicazione terapeutica, l'incidenza della neutropenia di grado severo, febbrile e non febbrile, risulta più elevata $(0,432)$ negli RCTs che prevedono la combinazione di olaparib e chemioterapia verso chemioterapia, ${ }^{21}$ rispetto ad analoghe esperienze di ricerca volte a sperimentare olaparib in monoterapia verso placebo, che riportano valori di incidenza compresi tra lo 0,037 e lo $0,10 .{ }^{58,59}$ Pertanto, per quanto concerne l'incidenza della neutropenia di grado severo per olaparib, il modello di valutazione economico-sanitaria ha adottato un'ipotesi di ricerca sfavorevole a tale principio attivo.

In termini più generali, l'impiego dei PARPi in monoterapia o in combinazione con chemioterapia ( $\mathrm{e}$, in questo caso, con quali farmaci) nelle pazienti con $\mathrm{CO}$ ricorrente pretrattato, richiede la raccolta di ulteriori evidenze empiriche non solo relativamente all'efficacia e alla tossicità di tali opzioni terapeutiche, ${ }^{56} \mathrm{ma}$ anche ai costi che esse generano a carico del terzo pagante.

Di pari rilevanza si reputa la disponibilità di risultati aggiornati sulla sopravvivenza totale delle pazienti trattate 
con PARPi e sulla qualità di vita connessa al loro stato di salute (o utilità), ${ }^{18,19,56,60}$ rispetto alla quale la tollerabilità degli eventi avversi esercita un effetto non trascurabile. In particolare, il tema dell'utilità (e di riduzione delle disutilità causate dalle tossicità da PARPi, sia di pertinenza ematologica ma, riteniamo, anche di differente tipologia) assume particolare rilievo per quelle pazienti per le quali, a motivo di prognosi fortemente infausta, lo spazio terapeutico risulti fatalmente limitato. ${ }^{56}$

La valutazione economica realizzata si inserisce in un filone di ricerca di recente attivazione in Italia, ${ }^{55}$ dedicato all'indagine degli aspetti economici delle tossicità in ambito oncologico o comprensive di tale obiettivo di ricerca. ${ }^{30} \mathrm{~A}$ tale proposito, confrontando i costi unitari per la gestione delle tossicità ematopoietiche di grado severo affrontate nel presente manoscritto con quanto riportato dalle due recenti pubblicazioni relative al nostro Paese citate con riferimento all'analisi di sensibilità di scenario, ${ }^{30,55}$ emergono, come precedentemente evidenziato, differenze degne di nota. Riteniamo che tali differenze dipendano solo marginalmente dalle fonti utilizzate per la monetizzazione delle risorse sanitarie secondo la prospettiva del SSN, che appartengono a un novero limitato soprattutto per la valorizzazione dei fattori produttivi diversi dai farmaci, ma essenzialmente dall'opinione qualificata dei clinici, variabile per patologia oncologica ed esperienza relativa al riscontro di determinati eventi avversi.

Inoltre, si osserva che, sebbene i costi unitari comparati si riferiscano a diverse neoplasie, le linee-guida nazionali consultate, ${ }^{13}$ dalle quali sono state ottenute parte delle risorse sanitarie finalizzate alla gestione delle tossicità ematopoietiche oggetto di indagine, si concentrano sulla gravità dell'evento avverso anziché sulla tipologia della malattia oncologica sottostante; quest'ultima non pare, pertanto, spiegare le differenze riscontate.

Con riferimento alla determinazione di impatto budgetario secondo incidenza, si evidenzia come il risparmio aggregato a favore di olaparib rimanga positivo, ancorché variabile in termini assoluti, per ciascuna delle variazioni delle ipotesi di base indotte nell'analisi di sensibilità univariata.

Qualora, mantenendo la prospettiva del SSN e confermando la totalità delle ipotesi a supporto della determinazione di impatto budgetario, si sommasse il costo per la gestione delle tossicità ematopoietiche al costo annuale della terapia con olaparib e niraparib, determinato, al netto degli sconti di legge, in $€ 5.302,34$ e $€ 8.430,72$ mensili, ${ }^{47,48}$ rispettivamente, nell'analisi di base il risparmio aggregato annuale per il SSN raggiungerebbe $€ 22,64 \mathrm{~m}$ (anno 2018), €22,59m (anno 2019) ed €22,54m (anno 2020), pari a $€ 67,78 \mathrm{~m}$ nel triennio 2018-2020. L'indifferenza economica tra olarib e niraparib si otterrebbe solo assumendo uno sconto che si attestasse intorno al $40 \%$ del costo mensile di quest'ultimo.
Quali sono i principali limiti relativi alla valutazione economica effettuata?

Un primo limite attiene alla modalità di comparazione indiretta dell'incidenza delle diverse tossicità ematopoietiche indagate nella presente esperienza di ricerca.

Peraltro, la scelta dell'incidenza, che ha informato sia l'analisi di minimizzazione dei costi, sia la determinazione di impatto budgetario (supportando, per quest'ultima, la reiterazione triennale dei risultati di costo ricavati dall'albero decisionale caratterizzato da un unico ciclo, ancorché su una popolazione femminile in lieve diminuzione) è stata essenzialmente dettata dalla disponibilità, secondo tale dimensione epidemiologica, dei dati ricavati dalla meta-analisi ripetutamente citata. ${ }^{6}$

Un secondo limite attiene alla natura modellistica dello studio che, per definizione, include dati provenienti da diverse fonti, ${ }^{61}$ nonché alcune stime conseguenti a ipotesi di ricerca. Tale ultimo aspetto si riflette, fatalmente, anche sulla quantificazione delle risorse sanitarie dedicate al trattamento delle tossicità ematopoietiche indagate.

Un terzo limite, peraltro riscontrato anche in altri Paesi, ${ }^{62,63}$ concerne la trasferibilità alla normale pratica clinica, per vincoli istituzionali, organizzativi ed economici, di quanto suggerito dalle linee-guida e dalla letteratura a proposito della gestione delle tossicità ematopoietiche, con possibili riflessi sui contesti di erogazione delle prestazioni sanitarie diverse dai farmaci $\mathrm{e}$, in ultima analisi, sui rispettivi costi unitari.

Un quarto e ultimo limite concerne l'imperfetta comparabilità dei costi di gestione delle tossicità ematopoietiche sopra commentate, poste le differenti modalità seguite per la raccolta dei dati relativi alla quantificazione delle risorse sanitarie. Infatti, mentre nella presente ricerca si è privilegiata l'integrazione tra fonti di letteratura e opinione qualificata di clinico, negli studi italiani precedentemente citati sono state impiegate, rispettivamente, un sondaggio ${ }^{30}$ e la metodologia Delphi. ${ }^{55}$

Per la totalità dei limiti sopra evidenziati, si profila pertanto la necessità di corroborare i risultati ottenuti con successive esperienze di ricerca empiriche, e possibilmente comparative, tra olaparib e niraparib in tale indicazione terapeutica, realizzate su dati desunti dalle cartelle delle pazienti o da database di carattere amministrativo. ${ }^{55}$

In conclusione, fatti salvi i limiti precedentemente dettagliati, si ritiene che, per quanto attiene alla gestione delle tossicità ematopoietiche indagate con riferimento alla terapia del CO $B R C A+$ pretrattato recidivato, olaparib rappresenti, per il SSN, un programma sanitario economicamente più efficiente di niraparib.

\section{Declaration of Conflicting Interest}

CL is a consultant for AstraZeneca S.p.A. NAM is an employee of AstraZeneca S.p.A. FP declares no conflicting interest. 


\section{Funding}

This study was funded by AstraZeneca S.p.A.

\section{Supplemental material}

Supplemental material for this article is available online.

\section{ORCID iD}

Carlo Lazzaro iD https://orcid.org/0000-0003-4795-1834

\section{Bibliografia}

1. Associazione Italiana Oncologia Medica (AIOM), Associazione Italiana Registri Tumori (AIRTUM) e Fondazione AIOM. I numeri del cancro in Italia 2018. Brescia: Intermedia Editore, 2018. https://www.aiom. it/wp-content/uploads/2018/10/2018_NumeriCancrooperatori.pdf (Ultima data di accesso al sito: 16/04/2019).

2. Associazione Italiana Oncologia Medica(AIOM). Linee guida. Tumori dell'ovaio. Edizione 2015. Milano: AIOM, 2015. http://media.aiom.it/userfiles/files/doc/LG/2015_LG_AIOM Ovaio.pdf (Ultima data di accesso al sito: 16/04/2019).

3. Conklin CMJ and Gilks CB. Differential diagnosis and clinical relevance of ovarian carcinoma subtypes. Expert Rev Obstet Gynecol. 2013; 8(1): 67-82.

4. Associazione Italiana Oncologia Medica (AIOM), Società Italiana di Genetica Umana (SIGU), Società Italiana di Biochimica (SIBioC) e Società Italiana di Anatomia Patologica e Citodiagnostica (SIAPEC-IAP). Raccomandazioni per l'implementazione del test BRCA nei percorsi assistenziali e terapeutici delle pazienti con carcinoma ovarico. 8 Luglio 2015. https://docs.biomedia. net/SIGU/RaccomandazioniBRCA.pdf (Ultima data di accesso al sito: 16/04/2019).

5. Isabelle M, Moreel X, Gagné JP, et al. Investigation of PARP-1, PARP-2, and PARG interactomes by affinitypurification mass spectrometry. Proteome Sci. 2010; 8: 22.

6. Zhou JX, Feng LJ and Zhang X. Risk of severe hematologic toxicities in cancer patients treated with PARP inhibitors: a meta-analysis of randomized controlled trials. Drug Des Devel Ther. 2017; 11: 3009-3017.

7. Kim G, Ison G, McKee AE, et al. FDA approval summary: olaparib monotherapy in patients with deleterious germline BRCA-mutated advanced ovarian cancer treated with three or more lines of chemotherapy. Clin Cancer Res. 2015; 21(19): 4257-4261.

8. Food and Drug Administration. FDA approves olaparib for germline BRCA-mutated metastatic breast cancer. https://www.fda.gov/Drugs/InformationOnDrugs/ ApprovedDrugs/ucm623540.htm (Ultima data di accesso al sito: 16/04/2019).

9. Scott LJ. Niraparib: first global approval. Drugs. 2017; 77(9): 1029-1034.

10. Alecu I, Milenkova $T$ and Turner SR. Risk of severe hematologic toxicities in cancer patients treated with PARP inhibitors: results of monotherapy and combination therapy trials. Drug Des Devel Ther. 2018; 12: 347-348.

11. Patibandla NS and Monga DK. Niraparib - A promising drug with hematological toxicity. J Oncol Pharm Pract. 2018; 1078155218800156.
12. Zhong L, Tran AT, Tomasino T, et al. Cost-effectiveness of niraparib and olaparib as maintenance therapy for patients with platinum-sensitive recurrent ovarian cancer. J Manag Care Spec Pharm. 2018; 24(12): 1219-1228.

13. Associazione Italiana Oncologia Medica (AIOM). Linee guida. Gestione della tossicità emopoietica in oncologia. Edizione 2017. http://media.aiom.it/userfiles/files/doc/ LG/2017_LGAIOM_Toss_ematopoietica.pdf (Ultima data di accesso al sito: $1 \overline{6 / 04 / 2019) \text {. }}$

14. Institute for Clinical and Economic Review (ICER). Appendix E. Comparative Value Supplemental Information. In: Poly ADP-Ribose Polymerase (PARP) Inhibitors for Ovarian Cancer: Effectiveness \& Value. Final Evidence Report. September 28, 2017, pp. 106-125. https://icerreview.org/wp-content/uploads/2017/02/MWCEPAC_ OVARIAN_EVIDENCE_REPORT_08302017.pdf (Ultima data di accesso al sito: 16/04/2019).

15. Banca d'Italia. Tassi di cambio. https://tassidicambio. bancaditalia.it/dailyRates (Ultima data di accesso al sito: 21/02/2019).

16. National Cancer Institute, National Institutes of Health, U.S. Department of Health and Human Services. Common Terminology Criteria for Adverse Events (CTCAE) version 5.0: November 27, 2017. https://ctep.cancer. gov/protocolDevelopment/electronic_applications/docs/ CTCAE_v5_Quick_Reference_8.5x11.pdf (Ultima data di accesso al sito: 16/04/2019).

17. Sackeyfio A, Nussey F, Friedlander M, et al. Comparative efficacy and tolerability of the PARP inhibitors olaparib 300 $\mathrm{mg}$ tablets BID, niraparib $300 \mathrm{mg}$ capsules QD and rucaparib $600 \mathrm{mg}$ tablets BID as maintenance treatment in BRCAmutated (BRCAm) platinum-sensitive relapsed ovarian cancer (PSROC). Gynecol Oncol. 2018; 149 (Suppl 1): 43-44.

18. Drummond MF, Schulper MJ, Claxton K, et al. Methods for the economic evaluation of health care programmes. 4 th ed. Oxford, UK: Oxford University Press, 2015.

19. Neumann PJ, Ganiats TG, Russell LB, et al.(eds). CostEffectiveness in Health and Medicine. 2nd ed. New York, NY: Oxford University Press, 2016.

20. Briggs A, Schulper M and Claxton K. Decision modelling for health economic evaluation. Oxford, UK: Oxford University Press 2006.

21. Oza AM, Cibula D, Benzaquen AO, et al. Olaparib combined with chemotherapy for recurrent platinum-sensitive ovarian cancer: a randomised phase 2 trial. Lancet Oncol. 2015; 16(1): 87-97.

22. Mirza MR, Monk BJ, Herrstedt J, et al. Niraparib maintenance therapy in platinum-sensitive, recurrent ovarian cancer. N Engl J Med. 2016; 375(22): 2154-2164.

23. Sonnenberg FA and Beck JR. Markov models in medical decision making: a practical guide. Med Decis Making. 1993; 13(4): 322-339.

24. Sistema Statistico Nazionale - Istituto Nazionale di Statistica. Demo-Geodemo. Mappe, Popolazione, Statistiche Demografichedell'ISTAT.Tavoledimortalitàdellapopolazione residente. Ripartizione: Italia - Femmine - Anno: 2017. http ://demo.istat.it/tvm2016/index.php?lingua=ita(Ultimoaccesso al sito: 16/04/2019).

25. Azienda Sanitaria Locale di Vercelli. Analisi della Mortalità generale e per cause nell'ASL "VC" di Vercelli e nei 
Distretti. Anno 2011. http://www.epicentro.iss.it/problemi/ mortalita/pdf/REPORT_MORTALITA_ASL_VC_2014. pdf (Ultimo accesso al sito: 16/04/2019).

26. Gold MR, Siegel JE, Russel LB, et al. (eds). Costeffectiveness in health andmedicine. New York, NY: Oxford University Press 1996, p. 199.

27. Kaye SB, Lubinski J, Matulonis U, et al. Phase II, openlabel, randomized, multicenter study comparing the efficacy and safety of olaparib, a poly (ADP-ribose) polymerase inhibitor, and pegylated liposomal doxorubicin in patients with BRCA1 or BRCA2 mutations and recurrent ovarian cancer. J Clin Oncol. 2012; 30(4): 372-379.

28. Ledermann J, Harter P, Gourley C, et al. Olaparib maintenance therapy in platinum-sensitive relapsed ovarian cancer. N Engl J Med. 2012; 366(15): 1382-1392.

29. Pagano $M$ and Gauvreau K. (edizione italiana a cura di Angelillo I, Pavia M e Villari P). Fondamenti di biostatistica. 2nd ed. Napoli: Idelson-Gnocchi, 2003.

30. Lazzaro C, Barone C, Caprioni F, et al. An Italian costeffectiveness analysis of paclitaxel albumin (nab-paclitaxel) + gemcitabine vs gemcitabine alone for metastatic pancreatic cancer patients: the APICE study. Expert Rev Pharmacoecon Outcomes Res. 2018; 18(4): 435-446.

31. Kern WV, Cometta A, De Bock R, et al. Oral versus intravenous empirical antimicrobial therapy for fever in patients with granulocytopenia who are receiving cancer chemotherapy. International Antimicrobial Therapy Cooperative Group of the European Organization for Research and Treatment of Cancer. N Engl J Med. 1999; 341(5): 312-318.

32. Hughes WT, Armstrong D, Bodey GP, et al. 2002 guidelines for the use of antimicrobial agents in neutropenic patients with cancer. Clin Infect Dis. 2002; 34(6): 730-751.

33. Commissione Regionale del Farmaco della Regione EmiliaRomagna (ed.). Scheda di valutazione di: Complessi del ferro(III) per uso ev (saccarato e carbossimaltosio). Bologna: Regione Emilia-Romagna, 2015, p. 6.

34. Petrucci MT, Calabrese E, Levi A, et al. Cost of illness in patients with multiple myeloma in Italy: the CoMiM study. Tumori. 2013; 99(4): e193-202.

35. Cortinovis D, Beretta G, Piazza E, et al. Chemotherapyinduced anemia and oncologist perception on treatment: results of a web-based survey. Tumori. 2013; 99(1): 45-50.

36. Castaman G and Pieri L. Management of thrombocytopenia in cancer. Thromb Res. 2018; 164 (Suppl 1): S89-S93.

37. Osservatorio Nazionale sull'Impiego dei Medicinali (OsMed). L'uso dei farmaci in Italia. Rapporto nazionale 2017. Roma: Agenzia Italiana del Farmaco, 2018, p. 103. http://www.aifa. gov.it/sites/default/files/Rapporto_OsMed_2017_AIFA-acc. pdf (Ultimo accesso al sito: 16/04/2019).

38. Torrinomedica S.r.l. Ricerca farmaci in commercio. Pagina aggiornata il 10/08/2018. http://www.torrinomedica.it/ farmaci/ricercadbfarmaci/RisultatiIndiceFarmaciAuto.asp (Ultimo accesso al sito: 16/04/2019).

39. Ministero della Salute. Progetto Mattoni SSN. Pronto Soccorso e sistema 118. Proposta metodologica per la valutazione dei costi dell'emergenza. Roma: Ministero della Salute, 2007, p. 15. http://www.mattoni.salute.gov.it/ mattoni/documenti/11_Valutazione_costi_dell_emergenza. pdf (Ultimo accesso al sito: 16/04/2019).
40. Ministero della Salute. Decreto 18 ottobre 2012. Remunerazione prestazioni di assistenza ospedaliera per acuti, assistenza ospedaliera di riabilitazione e di lungodegenza post acuzie e di assistenza specialistica ambulatoriale. (13A00528). Supplemento Straordinario n. 8 alla Gazzetta Ufficiale n. 23, 28 Gennaio 2013, Serie generale, Allegato 3.

41. Conferenza Permanente per i Rapporti tra lo Stato le Regioni e le Province Autonome di Trento e Bolzano. Accordo interregionale per la compensazione della mobilità sanitaria. Versione in vigore per le attività degli anni 20142015 e 2016. Roma: Conferenza Permanente per i Rapporti tra lo Stato le Regioni e le Province Autonome di Trento e Bolzano, 2 Febbraio 2017.

42. Ministero della Salute. Rapporto annuale sull'attività di ricovero ospedaliero. Dati SDO 2016. Roma: Ministero della Salute, Maggio 2017. http://www.salute.gov.it/portale/ documentazione/p6_2_2_1.jsp?lingua $=$ italiano $\&$ id $=2651$ (Ultimo accesso al sito: $1 \overline{6} / 04 / 2019)$.

43. Provincia Autonoma di Bolzano. Assessorato alla sanità. Prestazioni di assistenza specialistica ambulatoriale e relative tariffe, elencate secondo le branche specialistiche. Codice prestazione 99.22. Bolzano: Provincia Autonoma di Bolzano, 2018.

44. Fattore G. Proposta di linee guida per la valutazione economica degli interventi sanitari in Italia. Pharmacoeconomics Ital Res Articles. 2009;11(2): 83-93.

45. Trueman P, Drummond $\mathrm{M}$ and Hutton J. Developing guidance for budget impact analysis. Pharmacoeconomics. 2001; 19(6): 609-621.

46. Sullivan SD, Mauskopf JA, Augustovski F, et al. Budget impact analysis-principles of good practice: report of the ISPOR 2012 Budget Impact Analysis Good Practice II Task Force. Value Health. 2014; 17(1): 5-14.

47. Agenzia Italiana del Farmaco. Determina 6 aprile 2016. Riclassificazione del medicinale per uso umano "Lynparza", ai sensi dell'art. 8, comma 10, della legge 24 dicembre1993, n. 537. (Determina n. 504/2016). Gazzetta Ufficiale della Repubblica Italiana, Serie Generale, n. 96, 26 Aprile 2016.

48. Agenzia Italiana del Farmaco. Determina 24 agosto 2018. Regime di rimborsabilità e prezzo del medicinale per uso umano "Zejula". (Determina n. 1362/2018). Gazzetta Ufficiale della Repubblica Italiana, Serie Generale, n. 219, 20 Settembre 2018.

49. Sistema Statistico Nazionale - Istituto Nazionale di Statistica. Demo-Geodemo. Mappe, Popolazione, Statistiche Demografiche dell'ISTAT. Previsioni regionali della popolazione residente per sesso, età e regione - base 1/1/2017. Ripartizione: Italia -Femmine - Anni: 2017-2020. http://demo. istat.it/previsioni2017/index.php?lingua=ita (Ultimo accesso al sito: 16/04/2019)

50. Ushijima K. Treatment for recurrent ovarian cancer-at first relapse. J Oncol. 2010; 2010: 497429.

51. Cegedim Italy, S.r.l. (ed.). Piano di Ricerche a supporto del Lancio di Olaparib (Fase Estensiva). Progetto 213126. Sesto San Giovanni: Cegedim, 5 May 2014.

52. AstraZeneca S.p.A. Elaborazione su dati AZ Fastnet platform. Piattaforma web di esecuzione test AstraZeneca. Basiglio: Astrazeneca, May 2018. http:/www.azfastnet.it/ (Ultimo accesso al sito: 16/04/2019). 
53. Briggs AH. Handling uncertainty in economic evaluation and presenting the results. In: Drummond $\mathrm{M}$ and McGuire A eds. Economic evaluation in health care. Merging theory with practice. Oxford, UK: Oxford University Press, 2001, pp. 172-214.

54. Briggs $\mathrm{AH}$ and Gray AM. Handling uncertainty when performing economic evaluation of health care interventions. Health Technol Assess. 1999; 3(2): 1-134.

55. Favaretto A, Grossi F, Morabito A, et al. Il costo degli eventi avversi associati ad afatinib, erlotinib e gefitinib nel trattamento del tumore del polmone non a piccole cellule con mutazione EGFR. Global \& Regional Health Technology Assessment. 2017; 4(1): e187-196.

56. Wiggans AJ, Cass GKS, Bryant A, et al. Poly(ADPribose) polymerase (PARP) inhibitors for the treatment of ovarian cancer (Review). Cochrane Database Syst Rev. 2015;(5):CD007929.

57. Glick HA, Doshi JA, Sonnad SS, et al. Economic evaluation in clinical trials. 2nd ed. Oxford, UK: Oxford University Press, 2017.

58. Gourley C, Friedlander M, Matulonis UA, et al. Clinically significant long-term maintenance treatment with olaparib in patients with platinum-sensitive relapsed serous ovarian cancer. J Clin Oncol. 2017;35: 15_suppl, 5533-5533.
59. Pujade-Lauraine E, Ledermann JA, Selle F, et al. Olaparib tablets as maintenance therapy in patients with platinum-sensitive, relapsed ovarian cancer and a BRCA1/2 mutation (SOLO2/ENGOT-Ov-21): a doubleblind, randomised, placebo-controlled, phase 3 trial. Lancet Oncol. 2017; 18(9): 1274-1284.

60. National Institute for Health and Clinical Excellence (NICE). Olaparib for maintenance treatment of relapsed, platinumsensitive, BRCA mutation-positive ovarian, fallopian tube and peritoneal cancer after response to second-line or subsequent platinum-based chemotherapy. Technology appraisal guidance [TA381], published date: 27/01/2016. https://www.nice.org. uk/guidance/ta381 (Ultimo accesso al sito: 16/04/1959).

61. Caro JJ, Briggs AH, Siebert U, et al. Modeling good research practices-overview: a report of the ISPOR-SMDM Modeling Good Research Practices Task Force-1. Value Health. 2012;15(6):796-803.

62. Cabana MD, Rand CS, Powe NR, et al. Why don't physicians follow clinical practice guidelines? A framework for improvement. JAMA. 1999; 282(15): 1458-1465.

63. Lugtenberg M, Zegers-van Schaick JM, Westert GP, et al. Why don't physicians adhere to guideline recommendations in practice? An analysis of barriers among Dutch general practitioners. Implement Sci. 2009; 4: 54. 